\title{
Photon splitting in a strongly magnetized, charge-asymmetric plasma
}

\author{
Chistyakov M.V. ${ }^{1, \star}$, Rumyantsev D.A. ${ }^{1, \star \star}$, and Shlenev D.M. ${ }^{1,2, \star \star \star}$ \\ ${ }^{1}$ Division of Theoretical Physics, Department of Physics, \\ Yaroslavl State P. G. Demidov University, Sovietskaya 14, \\ 150000 Yaroslavl, Russian Federation \\ ${ }^{2}$ Division of Physics, \\ A. F. Mozhaiskiy Space Military Academy, Yaroslavl Branch, \\ Moskovskiy Prosp. 28, 150001 Yaroslavl, Russian Federation
}

\begin{abstract}
The process of the photon splitting, $\gamma \rightarrow \gamma \gamma$, is investigated in the presence of strongly magnetized charge-asymmetric cold plasma. The dispersion properties of photons and the new polarization selection rules are obtained in such plasma. The absorption rate of the leading photon splitting channel are calculated with taking account of the photon dispersion and wave function renormalization. In addition, a comparison of the photon splitting and the Compton scattering processes is performed.
\end{abstract}

\section{Introduction}

The process of photon splitting into two photons is a prominent example of the external active medium influence on the reactions with elementary particles. Though it is forbidden in vacuum by charge conjugation symmetry of QED known as Furry's theorem, it becomes allowed in the presence of an external electromagnetic field and/or plasma. In spite of the rather long history of the investigation [15] (for a review see e.g. [6-8]) the process of photon splitting still attracts a great interest stimulated by its possible applications.

It is remarkable, that such an exotic process may lead to observable physical manifestations in astrophysical objects. Its principal effect is to degrade photon energies and thereby to soften the gamma-ray spectra from neutron stars. In particular, it is supposed, that the photon splitting could explain a peculiarities in the gamma-ray spectra of some radio pulsars [9].

There is one more interesting application of the process under consideration concerning the radio quiescence of soft gamma repeaters (SGRs) and anomalous X-ray pulsars (AXPs). Because the photon splitting has no threshold, high-energy photons propagating at very small angles to the magnetic field in the neutron star magnetospheres may split before reaching the threshold of the pair production. Therefore, this process could change the production efficiency of electron-positron pairs, required for a detectable radio emission [10-13].

\footnotetext{
^e-mail: mch@uniyar.ac.ru

$\star \star$ e-mail: rda@uniyar.ac.ru

$\star \star \star$ e-mail: allen caleb@rambler.ru
} 
The process discussed here also plays an important role in the model of the SGR burst $[14,15]$. Namely, a formation is assumed of a "magnetically trapped fireball" in the near magnetosphere of a magnetar with hot $e^{+} e^{-}$-photon plasma in a local thermodynamic equilibrium. Notice that in all of these astrophysical models the photon splitting process in the presence of strong magnetic field is considered in the environment which is not necessarily approximated by vacuum. Instead, the presence of considerably dense and hot electron-positron plasma is possible. This plasma could significantly change the photon splitting rate.

Note, that the scale of the magnetic field of the above objects may reach the critical value $B_{e}=$ $m^{2} / e \simeq 4.41 \times 10^{13} \mathrm{G}^{1}$ or even exceed it, $B \gg B_{e}[16]$.

There are two ways in which a magnetized plasma influences the process under consideration. On the one hand, it modifies the photon dispersion properties. On the other hand, it can change the process amplitude. The first effect has been studied previously in Refs. [2, 17]. It was shown (see [2]) that in the case of the cold weakly magnetized plasma the process kinematics and the polarization selection rules remain the same as in the magnetized vacuum if the electron density is not too large $\left(n_{e} \lesssim 10^{19} \mathrm{~cm}^{-3}\right)$. In Ref. [17], the probability of the photon splitting was calculated by including the plasma effects on the photon dispersion but using the process amplitude obtained in the weak magnetic field without plasma. It was found that the plasma influence in such an approach was negligibly small, except the very narrow region of the plasma and magnetic field parameter space.

The modification of the photon splitting amplitude in the presence of a magnetic field and plasma was considered in Refs. [18, 19] on the basis of the Euler-Heisenberg effective Lagrangian with taking account of the thermal corrections in the one-loop and two-loop approximation correspondingly. It was noted that in the low temperature limit the process $\gamma \rightarrow \gamma \gamma$ could compete with the other absorption reactions such as the Compton scattering process.

Another approach was applied in Ref. [20]. Using the expansion of the electron propagator over the magnetic field strength, the amplitude and the absorption coefficient of the photon splitting were calculated in the high energy limit. The main conclusion of the paper was that the plasma effect was negligible. However, the estimations of the photon splitting absorption coefficients in a magnetic field presented in the paper in the high energy limit were incorrect because these expressions were applicable only in the low energy approximations. Note that in Refs. [18-20] the effects of the photon dispersion in magnetized plasma were not taken into account. However, the situation may change significantly in the limit of a strong magnetic field and plasma.

The self-consistent investigation of the photon splitting/merging and the Compton scattering including modifications of both dispersion relations and the process amplitude in magnetized plasma is provided in the recent paper [8]. The case of charge-symmetric plasma was studied in Ref. [8], however, a very interesting case of charge-asymmetric plasma with non zero chemical potential was not investigated in that paper. From the point of of possible astrophysical applications, such situation is, in our opinion, more realistic, and it is realized, for example, in the surface layer of a neutron star, where the process $\gamma \rightarrow \gamma \gamma$ can play an important role in forming an emission spectrum in this region (see, for example, Ref. [21]).

\section{Photon dispersion in the magnetized medium}

We begin to consider the processes $\gamma \rightarrow \gamma \gamma$ and $e \gamma \rightarrow e \gamma$ with investigation of the photon dispersion properties. The propagation of an electromagnetic radiation in any active medium is conveniently described in terms of normal modes (eigenmodes). In turn, the polarization and dispersion properties of the normal modes are defined by the eigenvectors and eigenvalues of the polarization operator $\mathcal{P}_{\alpha \beta}$,

\footnotetext{
${ }^{1}$ We use the natural units $c=\hbar=k=1, m$ is the electron mass, $e>0$ is the elementary charge.
} 
respectively. In this case, it is convenient to decompose the tensor $\mathcal{P}_{\alpha \beta}$ in terms of the basis of 4vectors [22] constructed from the electromagnetic field tensor reduced to a dimensionless form, and the 4-momentum of the photon $q_{\alpha}$ :

$$
\begin{array}{r}
b_{\mu}^{(1)}=(\varphi q)_{\mu}, \quad b_{\mu}^{(2)}=(\tilde{\varphi} q)_{\mu}, \\
b_{\mu}^{(3)}=q^{2}(\Lambda q)_{\mu}-q_{\mu} q_{\perp}^{2}, \quad b_{\mu}^{(4)}=q_{\mu} .
\end{array}
$$

Wherein $\left(b^{(1)} b^{*(1)}\right)=-q_{\perp}^{2},\left(b^{(2)} b^{*(2)}\right)=-q_{\|}^{2},\left(b^{(3)} b^{*(3)}\right)=-q^{2} q_{\|}^{2} q_{\perp}^{2},\left(b^{(4)} b^{*(4)}\right)=q^{2}$.

On the other hand, the presence of plasma can change the set of eigenvectors of the polarization operator compared in the case of a pure magnetic field. Then, in the plasma rest frame, we can represent the tensor $\mathcal{P}_{\alpha \beta}$ as an expansion over new eigenvectors $r_{\alpha}^{(\lambda)}$ with eigenvalues $\varkappa^{(\lambda)}$ [23]:

$$
\begin{aligned}
& \mathcal{P}_{\alpha \beta}=\sum_{\lambda=1}^{3} \varkappa^{(\lambda)} \frac{r_{\alpha}^{(\lambda)}\left(r_{\beta}^{(\lambda)}\right)^{*}}{\left(r^{(\lambda)}\right)^{2}}, \\
& r_{\beta}^{(\lambda)}=\sum_{i=1}^{3} A_{i}^{(\lambda)} b_{\beta}^{(i)},
\end{aligned}
$$

where $A_{i}^{(\lambda)}$ are some complex coefficients.

Hereafter we use the following notations: the four-vectors with the indices $\perp$ and $\|$ belong to the Euclidean $\{1,2\}$ subspace and the Minkowski $\{0,3\}$ subspace correspondingly. Then for arbitrary 4-vectors $A_{\mu}, B_{\mu}$ one has

$$
\begin{aligned}
& A_{\perp}^{\mu}=\left(0, A_{1}, A_{2}, 0\right), \quad A_{\|}^{\mu}=\left(A_{0}, 0,0, A_{3}\right), \\
& (A B)_{\perp}=(A \Lambda B)=A_{1} B_{1}+A_{2} B_{2}, \\
& (A B)_{\|}=(A \widetilde{\Lambda} B)=A_{0} B_{0}-A_{3} B_{3},
\end{aligned}
$$

where the matrices $\Lambda_{\mu \nu}=(\varphi \varphi)_{\mu \nu}, \widetilde{\Lambda}_{\mu v}=(\tilde{\varphi} \tilde{\varphi})_{\mu v}$ are constructed with the dimensionless tensor of the external magnetic field, $\varphi_{\mu \nu}=F_{\mu \nu} / B$, and the dual tensor, $\tilde{\varphi}_{\mu v}=\frac{1}{2} \varepsilon_{\mu \nu \rho \sigma} \varphi^{\rho \sigma}$. The matrices $\Lambda_{\mu v}$ and $\widetilde{\Lambda}_{\mu v}$ are connected by the relation $\widetilde{\Lambda}^{\mu \nu}-\Lambda^{\mu \nu}=g^{\mu \nu}=\operatorname{diag}(1,-1,-1,-1)$, and play the roles of the metric tensors in the perpendicular $(\perp)$ and the parallel $(\|)$ subspaces respectively.

In the case of strongly magnetized cold plasma with the temperature $T$ and the chemical potential $\mu$, in the limits $T \ll \mu-m$ and $e B \gg m^{2}, \mu^{2}, T^{2}$, we obtain the following coefficients $A_{i}^{(\lambda)}$ in the decomposition (3) with an accuracy of $O\left(1 /(e B)^{2}\right)$ [22, 23]:

$$
\begin{aligned}
& A_{1}^{(1,3)}=\mp \sqrt{q_{\perp}^{4}+(6 \Delta N \omega)^{2} \frac{q^{2}}{q_{\|}^{2}}}-q_{\perp}^{2}, \\
& A_{2}^{(1,3)}=\mathrm{i} \frac{\Delta N k_{z} q_{\perp}^{2}}{2 e B \mathcal{D}\left(q_{\|}\right) q_{\|}^{2}}\left[ \pm \sqrt{q_{\perp}^{4}+(6 \Delta N \omega)^{2} \frac{q^{2}}{q_{\|}^{2}}}+q_{\perp}^{2}\right], \\
& A_{3}^{(1,3)}=-\mathrm{i} \frac{6 \Delta N \omega}{q_{\|}^{2}}, \quad A_{1}^{(2)}=-\mathrm{i} \frac{\Delta N k_{z}}{2 e B \mathcal{D}\left(q_{\|}\right)}, \quad A_{2}^{(2)}=1, \quad A_{3}^{(2)}=0,
\end{aligned}
$$

where

$$
\mathcal{D}\left(q_{\|}\right)=-\mathcal{J}\left(q_{\|}\right)-H\left(\frac{q_{\|}^{2}}{4 m^{2}}\right),
$$




$$
\begin{aligned}
& \mathcal{J}\left(q_{\|}\right)=-\frac{1}{2 \sqrt{z(1-z)}}\left(\arctan \left[\frac{v_{F}-v_{\phi}+z v_{F}\left(v_{\phi}^{2}-1\right)}{\left(v_{\phi}^{2}-1\right) \sqrt{z(1-z)}}\right]+\right. \\
& \left.+\arctan \left[\frac{v_{F}+v_{\phi}+z v_{F}\left(v_{\phi}^{2}-1\right)}{\left(v_{\phi}^{2}-1\right) \sqrt{z(1-z)}}\right]\right), \quad 0 \leqslant z \leqslant 1 \\
& \mathcal{J}\left(q_{\|}\right)=-\frac{1}{4 \sqrt{z(z-1)}}\left(\ln \left[\frac{v_{F}-v_{\phi}+\left(v_{\phi}^{2}-1\right)\left(z v_{F}-\sqrt{z(z-1)}\right)}{v_{F}-v_{\phi}+\left(v_{\phi}^{2}-1\right)\left(z v_{F}+\sqrt{z(z-1)}\right)}\right]+\right. \\
& \left.+\ln \left[\frac{v_{F}+v_{\phi}+\left(v_{\phi}^{2}-1\right)\left(z v_{F}-\sqrt{z(z-1)}\right)}{v_{F}+v_{\phi}+\left(v_{\phi}^{2}-1\right)\left(z v_{F}+\sqrt{z(z-1)}\right)}\right]\right)-\frac{i \pi \Theta\left(v_{F}\left|v_{\phi}\right|-1\right)}{2 \sqrt{z(z-1)}}, \quad z>1, \\
& z=\frac{q_{\|}^{2}}{4 m^{2}}, \quad v_{F}=\frac{\sqrt{\mu^{2}-m^{2}}}{\mu}, \quad v_{\phi}=\frac{\omega}{k_{z}}, \quad \Delta N=2 p_{F}=2 \sqrt{\mu^{2}-m^{2}},
\end{aligned}
$$

$\Theta(x)$ is the theta-function,

$$
\begin{aligned}
& H(z)=\frac{1}{\sqrt{z(1-z)}} \arctan \sqrt{\frac{z}{1-z}}-1, \quad 0 \leqslant z \leqslant 1, \\
& H(z)=-\frac{1}{2 \sqrt{z(z-1)}} \ln \frac{\sqrt{z}+\sqrt{z-1}}{\sqrt{z}-\sqrt{z-1}}-1+\frac{i \pi}{2 \sqrt{z(z-1)}}, \quad z>1 .
\end{aligned}
$$

As one can see from the decomposition of the eigenvectors (3) over the basis (1) with the coefficients (4), in the case $\mu \neq 0$ and in a strong magnetic field, the photons have an elliptical polarization. However, in the cold, almost degenerate, moderately relativistic plasma, $\Delta N /(2 m) \simeq v_{F} \ll 1$, and from Eqs. (4) we obtain that the physical polarization vectors of the photons are just the same as in the charge-symmetric magnetized plasma [8]:

$$
\varepsilon_{\alpha}^{(1)}(q)=\frac{(q \varphi)_{\alpha}}{\sqrt{q_{\perp}^{2}}}, \quad \varepsilon_{\alpha}^{(2)}(q)=\frac{(q \tilde{\varphi})_{\alpha}}{\sqrt{q_{\|}^{2}}}
$$

In this approximation, the corresponding eigenvalues have the form

$$
\begin{aligned}
& \varkappa^{(1)}=-\frac{\alpha}{3 \pi} q_{\perp}^{2}+O\left(\frac{1}{(e B)^{2}}\right), \\
& x^{(2)}=\frac{2 \alpha}{\pi} e B \mathcal{D}\left(q_{\|}\right)+O\left(\frac{1}{e B}\right), \\
& \varkappa^{(3)}=O\left(\frac{1}{(e B)^{2}}\right) .
\end{aligned}
$$


In Figs. 1 and 2, the dispersion law of the mode 2 photon in both strong magnetic field and cold magnetized plasma is depicted as the solution of the equation

$$
q^{2}-x^{(2)}=0
$$

for various values of the chemical potential, propagation angles and photon momenta.

Moreover, as can be seen from Fig. 1, in degenerate plasma there arises the shift of the $e^{+} e^{-}$paircreation threshold which is defined in a pure magnetic field by the relation $q_{\|}^{2}=4 \mathrm{~m}^{2}$. One can see that in the region $\left|v_{\phi}\right|>1 / v_{F}\left(\left|k_{z}\right|<2 p_{F}\right)$ the last terms in Eqs. (10) and (7) cancel each other and the only contribution to the imaginary part of $\varkappa^{(2)}(q)$ comes from the logarithm function in Eq. (7). It is the fact that leads to the shift of the pair creation threshold from $q_{\|}^{2}=4 m^{2}$ to

$$
q_{\|}^{2}=2\left(\mu^{2}-p_{F}\left|k_{z}\right|+\mu \sqrt{\left(p_{F}-\left|k_{z}\right|\right)^{2}+m^{2}}\right) .
$$

This result is in agreement with a simple kinematical analysis of the process $\gamma_{2} \rightarrow e^{+} e^{-}$in degenerate plasma. Indeed, using the energy and momentum conservation laws with obviuos conditions $E \geqslant \mu$ and $\left|p_{z}\right| \geqslant p_{F}$ for the electron we come to the result (15).

It follows from Eq. (6) and (9) that the eigenvalue of the polarization operator $\varkappa^{(2)}$ becomes large near the electron-positron pair production threshold (see Eq. (15) and Figs. 1 and 2). This means that the renormalization of the wave function for a photon of this polarization should be taken into account:

$$
\varepsilon_{\alpha}^{(2)}(q) \rightarrow \varepsilon_{\alpha}^{(2)}(q) \sqrt{Z_{2}}, \quad Z_{2}^{-1}=1-\frac{\partial \varkappa^{(2)}(q)}{\partial \omega^{2}} .
$$

It is natural to expect that the presence of plasma can change the selection rules for the polarizations suggested in Ref. [2]. As one can see from Eqs. (11)-(13), only the eigenvalue $\varkappa^{(2)}(q)$ is modified in charge-asymmetric cold plasma. It means that the dispersion law of the mode 1 photon is the same as in the magnetized vacuum, where its 4-momentum is space-like and the deviation from the vacuum dispersion law, $q^{2}=0$, is negligibly small. On the other hand, the dispersion properties of the mode 2 photon essentially differ from the ones in magnetized vacuum. In this case the dispersion law in the form of the relation between $q_{\|}^{2}$ and $q_{\perp}^{2}$ additionally depends on the angle between the magnetic field direction and the photon momentum $q_{\|}^{2}=q_{\|}^{2}\left(q_{\perp}^{2}, \theta\right)$.

One can see from Figs. 1 and 2, that in the presence of magnetized plasma there exists the kinematical region, where $q^{2}>0$ contrary to the case of a pure magnetic field. It is connected with the appearance of the plasma frequency in the presence of the real electrons which can be defined from the equation

$$
\omega_{p l}^{2}-\varkappa^{(2)}\left(\omega_{p l}, \mathbf{k} \rightarrow 0\right)=0 .
$$

These facts lead to new polarization selection rules: in the region $q^{2}>0$, a new photon splitting channel $\gamma_{2} \rightarrow \gamma_{1} \gamma_{1}$ forbidden in the magnetic field without plasma, is possible, while the splitting channels $\gamma_{1} \rightarrow \gamma_{2} \gamma_{2}$ and $\gamma_{1} \rightarrow \gamma_{1} \gamma_{2}$ allowed in the pure magnetic field, are forbidden. In the region $q^{2}<0$, polarization selection rules are the same ones as in the magnetized vacuum [2].

It should be noted that, in the limit of cold plasma, $T \ll \mu-m$, the photons with energies near $\omega \sim T \ll m$, will participate in the splitting. Thus, the "new" splitting channel, $\gamma_{2} \rightarrow \gamma_{1} \gamma_{1}$, will be dominating in our conditions (see also Sec. 4). 
The partial amplitude for the channel $\gamma_{2} \rightarrow \gamma_{1} \gamma_{1}$ could be obtained from the results of the papers $[7,8]$ by the substitution of the corresponding polarization vectors $(10)$, and it can be represented in the following form:

$$
\mathcal{M}_{2 \rightarrow 11}=i 4 \pi\left(\frac{\alpha}{\pi}\right)^{3 / 2} \frac{\left(q^{\prime} \varphi q^{\prime \prime}\right)\left(q^{\prime} \tilde{\varphi} q^{\prime \prime}\right)}{\left[q_{\|}^{2} q_{\perp}^{\prime 2} q_{\perp}^{\prime \prime 2}\right]^{1 / 2}} \mathcal{D}\left(q_{\|}\right)
$$

where $\mathcal{D}\left(q_{\|}\right)$is given by the formula (5).

\section{The probability of the photon splitting}

To analyze the efficiency of the process under consideration and to compare it with other competitive reactions we calculate the photon absorption rate due to the photon splitting which can be defined in the following way:

$$
\begin{aligned}
& W_{\lambda \rightarrow \lambda^{\prime} \lambda^{\prime \prime}}=\frac{g_{\lambda^{\prime} \lambda^{\prime \prime}}}{32 \pi^{2} \omega_{\lambda}} \int\left|\mathcal{M}_{\lambda \rightarrow \lambda^{\prime} \lambda^{\prime \prime}}\right|^{2} Z_{\lambda} Z_{\lambda^{\prime}} Z_{\lambda^{\prime \prime}}\left(1+f_{\omega^{\prime}}\right)\left(1+f_{\omega^{\prime \prime}}\right) \times \\
& \times \delta\left(\omega_{\lambda}(\mathbf{k})-\omega_{\lambda^{\prime}}\left(\mathbf{k}-\mathbf{k}^{\prime \prime}\right)-\omega_{\lambda^{\prime \prime}}\left(\mathbf{k}^{\prime \prime}\right)\right) \frac{d^{3} k^{\prime \prime}}{\omega_{\lambda^{\prime}} \omega_{\lambda^{\prime \prime}}},
\end{aligned}
$$

where $f_{\omega}=\left[e^{\omega / T}-1\right]^{-1}$ is the photons distribution function, the factor $g_{\lambda^{\prime} \lambda^{\prime \prime}}=1-(1 / 2) \delta_{\lambda^{\prime} \lambda^{\prime \prime}}$ is inserted to account for the possible identity of the final photons.

As it was found in Sec. 2, in the presence of strongly magnetized plasma the "new" photon splitting channel $\gamma_{2} \rightarrow \gamma_{1} \gamma_{1}$ forbidden in pure magnetic field, becomes open. According to Eqs. (18) and (19), the absorption rate of the channel $\gamma_{2} \rightarrow \gamma_{1} \gamma_{1}$ can be presented as

$$
W_{2 \rightarrow 11}=\frac{\alpha^{3}}{8 \pi^{2}} Z_{2} \mathcal{D}^{2}\left(q_{\|}\right) \frac{q_{\perp}^{2}}{\omega} \mathcal{F}\left(\sqrt{\frac{q_{\|}^{2}}{q_{\perp}^{2}}}\right) \Theta\left(q^{2}\right),
$$

where

$$
\begin{aligned}
& \mathcal{F}(z)=\frac{4 z^{3}}{\pi} \int_{1-z}^{1+z} d x \int_{0}^{y_{0}} d y \frac{y^{2} \sqrt{y_{0}^{2}-y^{2}}}{\left(x^{2}+z^{2} y^{2}\right)\left[(2-x)^{2}+z^{2} y^{2}\right]} \times \\
& \left\{\left(1+f_{\omega_{+}}\right)\left(1+f_{\omega-\omega_{+}}\right)+\left(\omega_{+} \rightarrow \omega_{-}\right)\right\}, \\
& \times \omega_{ \pm}=\frac{1}{2 z^{2}}\left[\omega\left(z^{2}-1+x\right) \pm k_{z} z^{2} \sqrt{y_{0}^{2}-y^{2}}\right] \\
& y_{0}=\frac{\sqrt{z^{2}-1}}{z^{2}} \sqrt{z^{2}-(1-x)^{2}}, \quad z=\sqrt{\frac{q_{\|}^{2}}{q_{\perp}^{2}}}
\end{aligned}
$$

If one can neglect the effect of the final photon stimulation emission $\left(f_{\omega^{\prime}}=f_{\omega^{\prime \prime}}=0\right)$, the expression for the function $\mathcal{F}(z)$ is considerably simplified:

$$
\mathcal{F}(z)=2 \ln z-1+z^{-2} .
$$


In Fig. 3, the absorption rate of the channel $\gamma_{2} \rightarrow \gamma_{1} \gamma_{1}$ as the function of the initial photon energy is depicted for the case of the photon propagation across the magnetic field direction, for the chemical potential of $1 \mathrm{MeV}$ and $0.75 \mathrm{MeV}$. One can see that the probability of the "new" channel, $\gamma_{2} \rightarrow \gamma_{1} \gamma_{1}$, decreases rapidly with the chemical potential decreasing. This is due to an increase of the kinematically allowed region $\left(q^{2}>0\right)$ for the channel under consideration leading to the growth of the process phase space volume with the chemical potential increasing.

The dependence of the $\gamma_{2} \rightarrow \gamma_{1} \gamma_{1}$ splitting rate on the angle between the initial photon momentum and the magnetic field direction, for chemical potential of $0.75 \mathrm{MeV}$ is depicted in Fig. 4.

It is interesting to note that the qualitative behavior of the probability in the case of a cold, almost degenerate plasma is similar to the case of hot charge-symmetric plasma considered in Ref. [8]. This result is likely due to the fact, that in the cases $T \ll \mu-m$ and $\mu=0$ and in the region of $q^{2}>0$, the photon dispersion law is qualitatively the same, whereas the quantitative result is determined, basically, by the value of the phase volume of the reaction.

\section{Photon scattering}

Compton scattering is another type of the photon reaction, competing with the photon splitting. The process of the photon scattering in a strongly magnetized plasma, in degenerate plasma $(T \ll \mu-$ $m$ ), when the initial photon propagates across the magnetic field, for different chemical potential values and with taking account of the photon dispersion and the wave function renormalization was investigated recently (see Ref. [24]). Here we generalize our results to the case of an arbitrary angle between the initial photon momenta and the magnetic field direction.

In the degenerate plasma $(T \ll \mu-m)$, the absorption rates have the following form ${ }^{2}$ :

$$
\begin{aligned}
W_{1 \rightarrow 1} & \simeq \frac{\alpha^{2}}{4 \pi} \frac{B_{e}}{B} \frac{\omega^{3}}{m^{2} v_{F}^{2}}\left\{\left(1-v_{F} n_{z}\right)^{2} \int_{t_{1}}^{t_{2}} d t t \mathcal{F}\left(\frac{\omega}{T}, t\right)+\left(n_{z} \rightarrow-n_{z}\right)\right\}, \\
W_{2 \rightarrow 1} & \simeq \frac{\alpha^{2}}{4 \pi} \frac{B_{e}}{B} \frac{q_{\perp}^{2}}{q_{\|}^{2}} \frac{\omega^{3}}{m^{2} v_{F}^{2}} Z_{2} \Theta\left(\omega-\omega_{p l}\right)\left\{\left(v_{F}-n_{z}\right)^{2} \int_{t_{1}}^{t_{2}} d t t \mathcal{F}\left(\frac{\omega}{T}, t\right)+\left(n_{z} \rightarrow-n_{z}\right)\right\}, \\
& W_{1 \rightarrow 2} \simeq \frac{\alpha^{2}}{4 \pi} \frac{B_{e}}{B} \frac{\omega^{3}}{m^{2}}\left\{\Theta\left(\frac{\mu}{m}\left(1-n_{z} v_{F}\right) \omega-\omega_{p l}\right) \int_{t_{1}^{*}}^{t_{2}^{*}} d t t \mathcal{F}\left(\frac{\omega}{T}, t\right) \times\right. \\
& \left.\times\left.\left(1+\frac{m^{2}}{p_{F}^{2}} t-\frac{n_{z}}{v_{F}}\right)^{2}\left(1-\frac{\varkappa^{(2)}\left(q^{\prime}\right)}{q_{\|}^{\prime 2}}\right)\right|_{q_{\|}^{\prime 2}=\omega^{2}\left[(1-t)^{2}-\left(n_{z}-t / v_{F}\right)^{2}\right], k_{z}^{\prime}=\omega\left(n_{z}-t / v_{F}\right)}+\left(n_{z} \rightarrow-n_{z}\right)\right\},
\end{aligned}
$$

\footnotetext{
${ }^{2}$ The details of calculations of the photon absorption rate for the channel $e \gamma_{2} \rightarrow e \gamma_{2}$ can be found in Ref. [23].
} 


$$
\begin{aligned}
& W_{2 \rightarrow 2} \simeq \frac{\alpha^{2}}{2 \pi} \frac{B}{B_{e}}\left(\frac{m}{\mu}\right)^{6} \frac{q_{\|}^{2}}{\omega v_{F}^{4}} Z_{2}\left\{\frac{\Theta\left(\frac{\mu}{m}\left(1-n_{z} v_{F}\right) \omega-\omega_{p l}\right)}{\left(1-n_{z} v_{F}\right)^{4}} \int_{t_{1}^{*}}^{t_{2}^{*}} d t t \mathcal{F}\left(\frac{\omega}{T}, t\right) \times\right. \\
& \times\left[v_{F}^{2}(1-t)^{2}-\left(n_{z} v_{F}-t\right)^{2}\right]\left[\left(1-\frac{q_{\perp}^{2}}{4 m^{2}} \frac{B_{e}}{B}-\frac{q_{\|}^{\prime 2}-\varkappa^{(2)}\left(q^{\prime}\right)}{4 m^{2}} \frac{B_{e}}{B}\right)^{2}+\right. \\
& +\frac{\mu^{2} \omega^{2}}{2 m^{4}}\left(\frac{B_{e}}{B}\right)^{2} \frac{q_{\perp}^{2}}{q_{\|}^{\prime 2}}\left(1-\frac{x^{(2)}\left(q^{\prime}\right)}{q_{\|}^{\prime 2}}\right) \frac{\left(1-n_{z} v_{F}\right)^{2}}{\left(1-n_{z}^{2}\right)^{4}} \times \\
& \left.\left.\times\left(1-n_{z}^{2}-t\left(1-\frac{n_{z}}{v_{F}}\right)-\frac{\mu^{2}}{m^{2}}\left(1-n_{z} v_{F}\right)^{2}\right)^{2}\right]\left.\right|_{q_{\|}^{\prime 2}=\omega^{2}\left[(1-t)^{2}-\left(n_{z}-t / v_{F}\right)^{2}\right], k_{z}^{\prime}=\omega\left(n_{z}-t / v_{F}\right)}+\left(n_{z} \rightarrow-n_{z}\right)\right\},
\end{aligned}
$$

where

$$
\begin{gathered}
\mathcal{F}(x, t)=[1-\exp (-x t)]^{-1}[1-\exp (-x(1-t))]^{-1} \\
t_{2,1}= \pm \frac{v_{F}}{1 \pm v_{F}}\left(1 \pm n_{z}\right), \quad t_{2,1}^{*}=-\frac{v_{F}}{1-v_{F}^{2}}\left(v_{F}-n_{z} \mp \sqrt{\left(1-v_{F} n_{z}\right)^{2}-\frac{\omega_{p l}^{2}}{\omega^{2}}\left(1-v_{F}^{2}\right)}\right) \\
n_{z}=v_{\phi}^{-1}=\frac{\sqrt{q_{\perp}^{2}}}{\omega} \cot \theta
\end{gathered}
$$

One can see from Eqs. (21)-(25), that the absorption coefficients have significantly different dependences on the magnetic field strength, the energy and the chemical potential for the 1-mode and 2-mode photons. On the one hand, the absorption rates $W_{1 \rightarrow 1}, W_{2 \rightarrow 1}$ and $W_{1 \rightarrow 2}$ are inversely proportional to the magnitude of the magnetic field and strongly depend on the initial photon frequency to give a ground to expect the efficient diffusion of the 1-mode photon, as it takes place in hot chargesymmetric plasma case [8]. On the other hand, one can see that the absorption rate $W_{2 \rightarrow 2}$ is directly proportional to the magnetic field value and strongly depends on the chemical potential in contrast to the other channels. It is interesting to note that in the emission region with smaller $\mu^{3}$ it is seen that the large value of $W_{2 \rightarrow 2}$ and its weak dependence on the energy could lead to the trapping of the 2-mode. Therefore, such photons quickly come into the equilibrium with medium. Thus, the mode-1 photons are interesting for the not-blackbody spectrum formation.

Previous investigations of the radiation transfer problem in strongly magnetized plasma have shown that along with the Compton scattering process, the photon splitting $\gamma \rightarrow \gamma \gamma$ could play a significant role as a mechanism of the photon production $[8,14]$. As it was noted earlier (see also Sec. 3), in our conditions the major channels of the mode 2 photon conversion will be the splitting $\gamma_{2} \rightarrow \gamma_{1} \gamma_{1}$ and the scattering $e \gamma_{2} \rightarrow e \gamma_{1}$. To compare these processes, we have estimated the probability of the photon splitting and the Compton scattering rates near $\omega \sim \omega_{p l} \simeq \sqrt{(2 \alpha e B / \pi) v_{F}}$ and for nonrelativistic cold plasma $\left(v_{F} \ll 1\right)$ in the following way

$$
\begin{aligned}
& W_{2 \rightarrow 11} \simeq \frac{\alpha^{3} v_{F}^{2}}{8 \pi^{2}} \omega_{p l}\left(1-\frac{\omega_{p l}^{2}}{\omega^{2}}\right) \sin ^{2} \theta \times \\
& \times\left\{-\ln \left[\left(1-\frac{\omega_{p l}^{2}}{\omega^{2}}\right) \sin ^{2} \theta\right]-\cos ^{2} \theta-\frac{\omega_{p l}^{2}}{\omega^{2}} \sin ^{2} \theta\right\} \frac{\Theta\left(\omega-\omega_{p l}\right)}{\left(1-\mathrm{e}^{-\omega_{p l} /(2 T)}\right)^{2}},
\end{aligned}
$$

\footnotetext{
${ }^{3}$ This situation is realized, for example, in the surface layer of a neutron star with the density $\rho \sim 10^{6} \mathrm{~g} / \mathrm{cm}^{3}$.
} 


$$
W_{2 \rightarrow 1} \simeq \frac{2 \alpha^{3} v_{F}^{2}}{\pi^{2}} T\left(1-\frac{\omega_{p l}^{2}}{\omega^{2}}\right) \sin ^{2} \theta \frac{\Theta\left(\omega-\omega_{p l}\right)}{1-\mathrm{e}^{-\omega_{p l} / T}} .
$$

It follows from Eqs. (25) and (26), that $W_{2 \rightarrow 11} \sim W_{2 \rightarrow 1}$ in some region of the plasma parameters. Thus, we can see now that in the presence of cold plasma the process of the photon splitting could not be the only intensive source of the photon production but also an effective absorption mechanism. Therefore, the both processes of the photon splitting and the photon scattering are important for the $\mathrm{X}$-ray spectra formation.

\section{Conclusion}

The obtained results show that the plasma influence modifies the polarization selection rules in comparison with a pure magnetic field. In particular, the "new" splitting channel $\gamma_{2} \rightarrow \gamma_{1} \gamma_{1}$ which is forbidden without plasma, is allowed in the plasma presence.

On the other hand, the presence of plasma suppresses the probabilities of the channels $\gamma_{1} \rightarrow \gamma_{1} \gamma_{2}$ and $\gamma_{1} \rightarrow \gamma_{2} \gamma_{2}$ in comparison with a pure magnetic field.

The comparison of the photon splitting process and the Compton scattering shows that the influences of these reactions on the 1-mode radiation transfer are competitive in the cold plasma $(T \ll \mu-m)$. As a result, it could lead to the modification of the mechanism of the emission spectra formation of neutron stars.

\section{Acknowledgements}

We are grateful to A. V. Kuznetsov for useful discussions and valuable remarks. D. R. expresses his deep gratitude to the organizers of the Seminar "Quarks-2016" for warm hospitality.

The study was supported in part by the Russian Foundation for Basic Research (Project No. 14-02-00233-a). The work of D.R. was performed with the support by the Project No. 984 within the base part of the State Assignment for the Yaroslavl University Scientific Research.

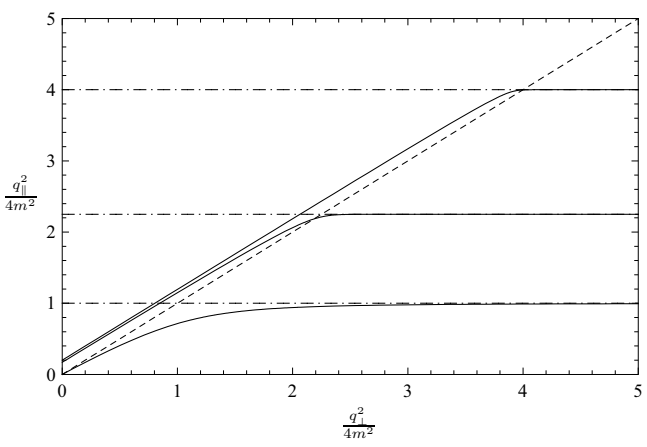

Figure 1. The photon dispersion in a strong magnetic field $\left(B / B_{e}=200\right)$ and degenerate $(T=0)$ plasma vs. chemical potential $\mu=1 \mathrm{MeV}$ (upper curve), $\mu=0.75 \mathrm{MeV}$ (middle curve) and $\mu=0.5 \mathrm{MeV}$ (lowest curve). The dotted line corresponds to the vacuum dispersion law, $q^{2}=0$. The angle between the photon momentum and the magnetic field direction is $\pi / 2$. 


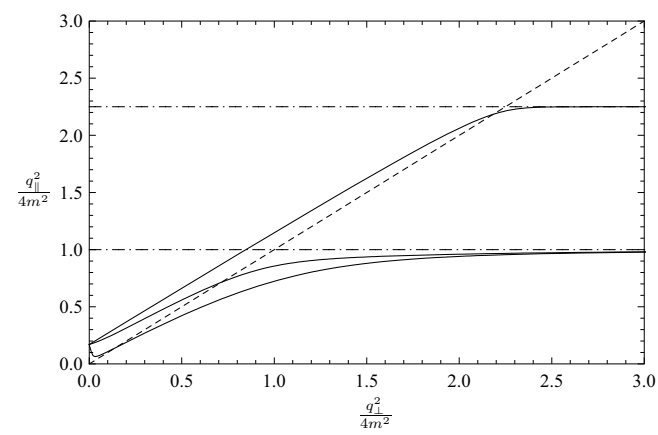

Figure 2. The photon dispersion in a strong magnetic field $\left(B / B_{e}=200\right)$ and degenerate plasma at $\mu=0.75 \mathrm{MeV}$ vs. the angle between the photon momentum and the magnetic field direction $\theta=\pi / 2$ (upper curve), $\theta=\pi / 4$ (middle curve) and $\theta=\pi / 12$ (lowest curve). The dotted line corresponds to the vacuum dispersion law, $q^{2}=0$.

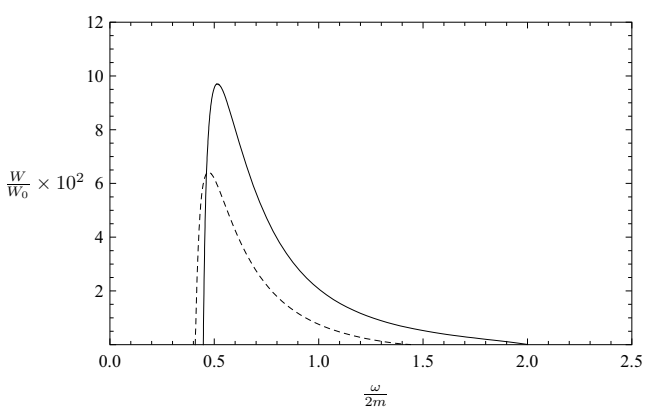

Figure 3. The photon absorption rate due to the channel $\gamma_{2} \rightarrow \gamma_{1} \gamma_{1}$ in a strong magnetic field $\left(B / B_{e}=200\right)$ and cold plasma $(T=50 \mathrm{keV})$ at chemical potential $1 \mathrm{MeV}$ (solid line) and $0.75 \mathrm{Mev}$ (dashed line). Here, $\theta=\pi / 2$, $W_{0}=(\alpha / \pi)^{3} m \simeq 3.25 \cdot 10^{2} \mathrm{~cm}^{-1}$.

\section{References}

[1] Z. Bialynicka-Birula, L. I. Bialynicka-Birula, Phys. Rev. D 2, 2341 (1970).

[2] S. L. Adler, Ann. Phys. (N.Y.) 67, 599 (1971).

[3] V. O. Papanian, V. I. Ritus, Sov. Phys. JETP 34, 1195 (1972).

[4] M. V. Chistyakov, A. V. Kuznetsov, N. V. Mikheev, Phys.Lett. B434, 67 (1998).

[5] J. I. Weise, Phys. Rev. D 69, 105017 (2004).

[6] V. O. Papanian, V. I. Ritus: Three-Photon Interaction in an Intense Field. In: Issues in IntenseField Quantum Electrodynamics, ed. by V.L. Ginzburg (Nova Science Publishers, New York, 1989) pp. 153-179.

[7] D. A. Rumyantsev, M. V. Chistyakov, JETP 101, 635 (2005).

[8] D. A. Rumyantsev, M. V. Chistyakov, N. S. Stus' Phys. Rev. D 86, 043007 (2012).

[9] A. K. Harding, M. G. Baring, P. L. Gonthier, Astrophys.J. 476, 246 (1997).

[10] M. G. Baring, Astrophys. J. 440, L69 (1995).

[11] M. G. Baring, A. K. Harding, Astrophys. J. Lett. 507, L55 (1998).

[12] V. M. Malofeev, O. I. Malov, D. A. Teplykh, et al., Astron. Rep., 49, 242 (2005). 


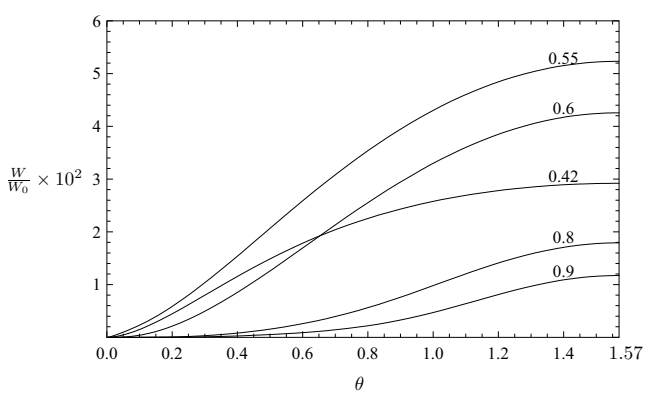

Figure 4. The dependence of the absorption rate of the channel $\gamma_{2} \rightarrow \gamma_{1} \gamma_{1}$ on the angle between the initial photon momentum and the magnetic field direction at different initial photon energies $\left(B / B_{e}=200, T=50 \mathrm{keV}\right.$, $\mu=0.75 \mathrm{MeV})$. The numbers above the curves correspond to the values of the ratio $\omega / 2 \mathrm{~m}$.

[13] Y. N. Istomin, D. N. Sobyanin, Astronomy Letters, 33, 660 (2007).

[14] C. Thompson, R. C. Duncan, Mon. Not. Roy. Astron. Soc. 275, 255 (1995).

[15] C. Thompson, R. C. Duncan, Astrophys. J. 561, 980 (2001).

[16] R. C. Duncan, C. Thompson, Astrophys. J. 392, L9 (1992).

[17] T. Bulik, Acta Astronomica. 48, 695 (1998).

[18] P. Elmfors, B. Skagerstam, Phys. Lett. B427, 197 (1998).

[19] H. Gies, Phys. Rev. D61, 085021 (2000).

[20] J. M. Martinez Resco, M. A. Valle Basagoiti, Phys. Rev. D64, 016006 (2001).

[21] V. Suleimanov, J. Poutanen, K. Werner, Astronomy \& Astrophysics. 545, A120 (2012).

[22] A. E. Shabad, Tr. Fiz. Inst. Akad. Nauk SSSR 192, 5 (1988).

[23] N. V. Mikheev, D. A. Rumyantsev, M. V. Chistyakov, Zh. Eksp. Teor. Fiz. 146, 289 (2014) [J. Exp. Theor. Phys. 119, 251 (2014)].

[24] D. A. Rumyantsev, M. V. Chistyakov, Int. J. Mod. Phys. A 24, 3995 (2009). 